\title{
Sentinel Nodes in Cervical Cancer: Surgical Innovation Outside the Ivory Towers
}

\author{
Michael Frumovitz and Pedro T. Ramirez \\ Department of Gynecologic Oncology and Reproductive Medicine, MD Anderson Cancer Center, Houston, TX
}

Lymphatic mapping and sentinel lymph node biopsy have long been established as 'standard of care' in the treatment of malignant melanoma and breast cancer. More recently, large prospective studies have shown its utility in the treatment of women with vulvar cancer, ${ }^{1,2}$ and many gynecologic oncologists have learned the technique for treating these patients. As we have become comfortable performing lymphatic mapping in women with vulvar cancer, we have started exploring the procedure in women with uterine and cervical cancers.

In theory, cervical cancer is an ideal malignancy for employing lymphatic mapping and sentinel node biopsy. First, the cervix is an 'external' organ, easily accessible for injection to perform preoperative imaging studies such as lymphoscintigraphy or single-photon emission computed tomography/computed tomography (SPECT/CT). Next, only $15-20 \%$ of women with operable cervical cancer (stages IA2-IIA) will have positive pelvic lymph nodes, meaning $80-85 \%$ will be exposed to the morbidities of complete pelvic lymphadenectomy without any benefit. Third, the cervix is a midline organ with fairly reliable drainage to the bilateral pelvic nodes, all of which are easily visualized with a single open incision or through minimally invasive surgery. However, $5 \%$ of women have aberrant drainage to the presacral or low para-aortic lymph nodes, ${ }^{3}$ making identification of these basins of great potential benefit as they are typically not part of the standard node dissection for this disease. Finally, the less radical, more precise nature of sentinel lymph node biopsy nicely compliments our field's move towards more

\footnotetext{
(C) Society of Surgical Oncology 2014
}

First Received: 3 November 2014; Published Online: 3 December 2014

M. Frumovitz

e-mail: mfrumovitz@mdanderson.org minimally invasive approaches for treating women with gynecologic malignancies.

However, in reality, gynecologic oncologists are still grappling with whether to adopt sentinel lymph node biopsy as standard of care for women with cervical cancer. Unlike the high incidence of melanoma (76,100 new cases/ year) or breast cancer $(235,030$ new cases/year), cervical cancer is a rare cancer in the developed world with only 12,360 new cases in the US this year, ${ }^{4}$ of which less than half will be operable candidates. Due to the rarity of the disease, large, prospective studies to prove its safety and utility have been difficult to perform. Although two multicenter studies have been performed in Europe with mixed results, ${ }^{5,6}$ most of our data regarding sentinel node biopsy for treating women with cervical cancer have come from single-institution studies.

In this month's issue of Annals of Surgical Oncology, Freitas and colleagues present their data on 57 patients with early-stage cervical cancer who underwent lymphatic mapping and sentinel node biopsy followed by complete pelvic lymphadenectomy. They found a sentinel node detection rate of $84 \%$, with bilaterality in $58 \%$ of these cases. Overall, the sensitivity of the procedure was $89 \%$, with a negative predictive value of $98 \%$. Sentinel node detection rate, bilaterality, sensitivity, and negative predictive value were similar to most other reports in the literature. ${ }^{7}$

We would expect bilateral drainage from the cervix, yet only $60-80 \%$ of cases that successfully map have bilateral sentinel nodes, meaning 20-40\% have only unilateral drainage. To account for the lack of bilateral drainage in this minority of patients, an algorithm developed at Memorial Sloan Kettering Cancer Center has gained popularity and acceptance within our field. ${ }^{8}$ In this algorithm, if a hemi-pelvis does not map, complete lymphadenectomy is performed. In the study by Freitas et al., the lone patient with a false negative had a positive pelvic node in a hemi- 
pelvis with no sentinel nodes detected. Applying this algorithm to their data would have detected all patients with lymph node metastases and therefore not undertreated any patient adjuvantly.

The fact that the investigators were able to achieve success similar to previous reports in the literature is of major importance as this study was performed in Brazil, a developing country, where resources and technology may be scarce. Virtually all of the previous studies evaluating sentinel nodes in cervical cancer were performed in the US or Europe where cervical cancer is rare. In stark contrast, the developing world continues to deal with cervical cancer as a major cause of cancer mortality, ranking second in cancer-related death among women. Showing that these techniques can be accurately performed in the areas of the world where cervical cancer remains a major public health concern, as these authors show, is particularly notable.

Furthermore, the results reported here might have been even better had the investigators not included patients with large tumors. In this study, $25 \%$ of patients had stage IB2 disease-tumors $>4 \mathrm{~cm}$ in size but limited to the cervix. Overall, the detection rate in tumors $>2 \mathrm{~cm}$ is $80 \%$ compared with a detection rate of $95 \%$ for tumors $<2 \mathrm{~cm}$ in size. ${ }^{7}$ This phenomenon is likely due to difficulty injecting at the interface of the tumor mass and normal tissue, possible increased debris in lymphatic channels from large, necrotic tumors, and higher rates of bulky positive nodes as the tumor size increases.

Often, advances in technology and therapeutics only benefit those patients who can be treated at the academic, tertiary care centers that develop and pioneer them. In the next decade, we expect continued increase in the incorporation of lymphatic mapping and sentinel nodes in the treatment of women with cervical cancer. As the developing world will continue to see the vast majority of women with cervical cancer, it is reassuring to know that these techniques will be available for patients and surgeons worldwide.

\section{REFERENCES}

1. Van der Zee AG, Oonk MH, De Hullu JA, Ansink AC, Vergote I, Verheijen RH, et al. Sentinel node dissection is safe in the treatment of early-stage vulvar cancer. J Clin Oncol. 2008;26:8849

2. Levenback CF, Ali S, Coleman RL, Gold MA, Fowler JM, Judson $\mathrm{PL}$, et al. Lymphatic mapping and sentinel lymph node biopsy in women with squamous cell carcinoma of the vulva: a gynecologic oncology group study. J Clin Oncol. 2012;30:3786-91.

3. Marnitz S, Kohler C, Bongardt S, Braig U, Hertel H, Schneider A; German Association of Gynecologic Oncologists (AGO). Topographic distribution of sentinel lymph nodes in patients with cervical cancer. Gynecol Oncol. 2006;103:35-44.

4. American Cancer Society. Cancer facts and figures 2014. Atlanta (GA): American Cancer Society; 2014.

5. Altgassen C, Hertel H, Brandstadt A, Kohler C, Durst M, Schneider A; AGO Study Group. Multicenter validation study of the sentinel lymph node concept in cervical cancer: AGO Study Group. J Clin Oncol. 2008;26:2943-51.

6. Lecuru F, Mathevet P, Querleu D, Leblanc E, Morice P, Darai E, et al. Bilateral negative sentinel nodes accurately predict absence of lymph node metastasis in early cervical cancer: results of the SENTICOL study. J Clin Oncol. 2011;29:1686-91.

7. Rob L, Robova H, Jiri HM, Martin H, Petr S. Current status of sentinel lymph node mapping in the management of cervical cancer. Expert Rev Anticancer Ther. 2013;13:861-70.

8. Cormier B, Diaz JP, Shih K, Sampson RM, Sonoda Y, Park KJ, et al. Establishing a sentinel lymph node mapping algorithm for the treatment of early cervical cancer. Gynecol Oncol. 2011;122: 275-80. 